\section{Venöse Thromboembolie}

\section{Edoxaban ist Heparin nicht unterlegen}

Für Patienten mit Tumorerkrankungen und venösem thromboembolischen Ereignis (VTE) empfehlen die Leitlinien für die Rezidivprophylaxe niedermolekulares Heparin. Über die langfristige Effektivität und Sicherheit direkter oraler Antikoagulanzien (DOAC) lagen bisher keine Untersuchungen vor. Die internationale HOKUSAI-Studie verglich deshalb Edoxaban und subkutanes Dalteparin bei Patienten mit Karzinom und thromboembolischer Komplikation.

An HOKUSAI nahmen 114 Zentren in 13 Ländern teil. Insgesamt wurden 1050 Patienten randomisiert. Überwiegend bestand eine aktive Tumorerkrankung, jedes zweite Karzinom war metastasiert und etwa jeder fünfte Patient hatte $\geq 3$ Risikofaktoren für eine Blutung. 522 Erkrankte erhielten nach einer $\geq 5$-tägigen Startphase mit niedermolekularem Heparin

täglich $60 \mathrm{mg}$ orales Edoxaban (211 Tage median) und 524 Patienten bekamen subkutanes Dalteparin (184 Tage median). Bei einer Niereninsuffizienz erfolgte eine Dosisreduktion in Abhängigkeit von der glomerulären Filtrationsrate. Hauptendpunkt der Studie war das Kompositum aus VTE-Rezidiv und schwerer Blutung. Eine bedrohliche Hämorrhagie

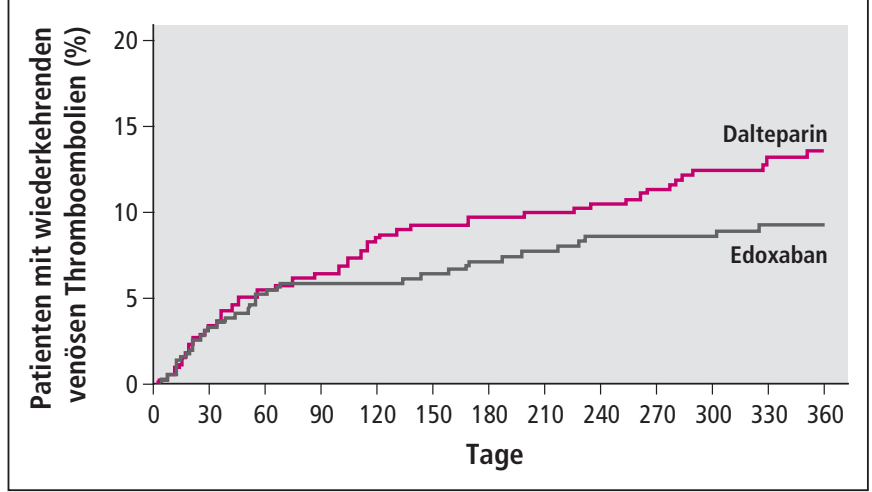

Abb. 1

Wiederkehrende Tumor-assoziierte venöse Thromboembolien unter Dalteparin und Edoxaban

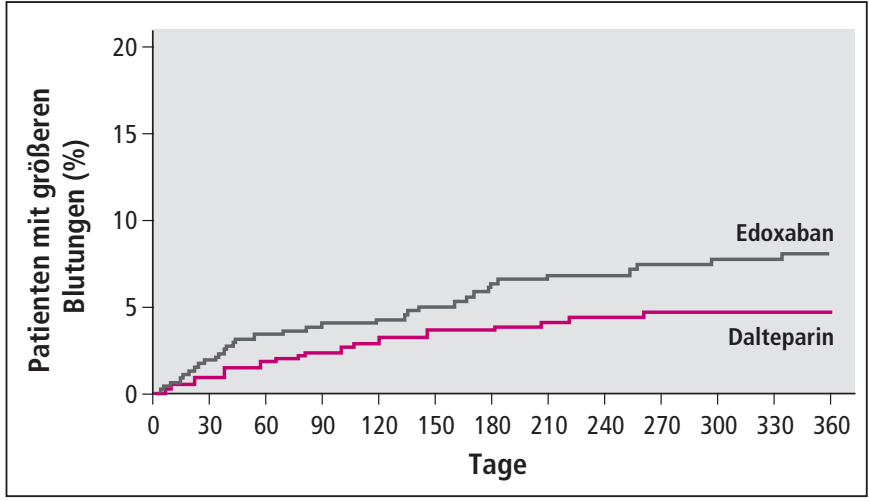

Abb. 2

Patienten mit größeren Blutungen unter Dalteparin und Edoxaban

\section{Fazit für die Praxis}

Insgesamt reduzierte Edoxaban das Rezidivrisiko um den Preis häufigerer relevanter Blutungen. Deutsche Fachgesellschaften raten zur individuellen Risikoabwägung unter Berücksichtigung der Tumorentität, des Alters, Allgemeinzustandes und anderer Tumor- und Patientencharakteristika.

kennzeichnete ein Hämoglobinabfall $>2 \mathrm{~g} / \mathrm{dl}$, ein Transfusionsbedarf von $\geq 2$ Konserven, eine kritische Blutungslokalisation und der blutungsassoziierte Tod.

Der primäre Endpunkt trat bei 67 Patienten (Edoxaban 12,8\%) und 71 Patienten (Dalteparin 13,5\%) ein. Die Nichtunterlegenheit des Faktor-Xa-Inhibitors war signifikant $(p=0,006)$. Eine Überlegenheit bestätigte sich nicht. In der Edoxaban- und Dalteparingruppe traten erneute VTE/schwere Hämorrhagien bevorzugt in den sechs Monaten nach dem Erstereignis auf (55 und 56 Patienten).

In der Einzelanalyse der Variablen reduzierte Edoxaban das Rezidivrisiko stärker als Dalteparin (Hazard Ratio [HR 0,71; $95 \%$ Konfidenzintervall $[K I$ 0,48-1,06; $p=0,09)$. $>A b-$ bildung 1 zeigt die unterschiedliche Wahrscheinlichkeit ab dem dritten Behandlungsmonat. Dem stand ein erhöhtes Blutungsrisiko gegenüber. Verglichen mit $4 \%$ der Dalteparingruppe kamen bei 6,9\% der Edoxabangruppe schwere Blutungen vor (HR 1,77; $95 \%-\mathrm{KI}$ 1,03-3,04; $p=0,04)$. Abbildung 2 verdeutlicht das größere Risiko ab dem Therapiestart und den wachsenden Unterschied. Die Differenz entstand vorwiegend durch die höhere Rate oberer gastrointestinaler Blutungen, von denen überwiegend Patienten mit gastrointestinalen Tumoren betroffen waren.

Dr. med. Susanne Krome, Melle

\section{Literatur}

1. Raskob GE et al. Edoxaban for the Treatment of Cancer-Associated Venous Thromboembolism. N Engl J Med 2017; doi:10.1056/NEJMoa1711948. 